\title{
Direct Policy Search for Multiobjective Optimization of the Sizing and Operation of Citizen Energy Communities
}

\author{
Armin Golla \\ Karlsruhe Institute of Technology \\ armin.golla@kit.edu \\ Philipp Staudt \\ Karlsruhe Institute of Technology \\ philipp.staudt@kit.edu
}

\author{
Robin-Joshua Meinke \\ Karlsruhe Institute of Technology \\ robin-joshua.meinke@kit.edu \\ C. Lindsay Anderson \\ Cornell University \\ cla28@cornell.edu
}

\author{
M. Vivienne Liu \\ Cornell University \\ m12589@cornell.edu \\ Christof Weinhardt \\ Karlsruhe Institute of Technology \\ weinhardt@kit.edu
}

\begin{abstract}
Citizen Energy Communities (CECs) are increasingly promoted in the European Union and beyond as a form of locally operated microgrids. While traditional microgrid research is often focused on an optimized operation, individual preferences regarding conflicting objectives are becoming more important in such communities. In this study, we present an evolutionary algorithm that has previously been used for a multiobjective operation of microgrids and include the perspective of heat consumption and initial sizing decisions using direct policy search. This way, the developed tool can be used by CEC planners to integrate conflicting objectives of residents in the installation phase. We introduce the algorithm formulation and demonstrate its functionality on a case study for different ambient conditions. The results show the opportunities to size and operate CECs through the presented algorithm.
\end{abstract}

\section{Introduction}

The international transition to more renewable energy sources and the associated subsidy schemes as well as the cost degradation of household sized renewable generation capacity, especially solar photovoltaics (PV), lead to an increased power generation on a household level [1]. Such small scale generation was originally a solution for microgrids that would sustain service in case of an outage for small communities or serve remote or island communities [2]. The increasing electrification of the transport and heat sector as well as the availability of residential battery solutions increase the ability of such microgrid communities to supply themselves with electricity and allow them to become increasingly independent of the transmission grid [3].
Currently, operating such microgrids is a challenge as multiple, sometimes conflicting objectives of the microgrid community need to be considered [4]. Furthermore, the optimal operation of battery storage given uncertain generation and demand is a constant subject of research [5]. First approaches to the operation of microgrids have been published [6]. The authors are mostly focusing on an optimization of the available resources subject to one objective function. Recently, a study emerged that focuses on multiple objectives in a microgrid using evolutionary algorithms, presenting only non-dominated strategies [4]. However, with an increasing interest in microgrid communities, specifically in the European Union under the term Citizen Energy Communities (CEC) [7], it becomes more important to not only focus on the operation but also the installation of such microgrids and to take different objectives and individual preferences such as financial considerations or local carbon emissions into account. Therefore, in this paper, we apply the Borg multi-objective evolutionary algorithm (MOEA) [8], that is used to co-optimize conflicting objectives, to integrate the decisions on the installation of resources and the decisions within the operation strategy with regards to multiple objectives into one problem. As a result, we are able to provide microgrid stakeholders with multiple possible courses of action based on non-dominated strategies. With this, we contribute to the further development of applications for evolutionary algorithms and we provide a practical tool for planners and residents to design microgrids within their premises. We thus answer the following research questions:

1. How can competing objectives in regards to the installation and operation of a community microgrid be optimized through an evolutionary algorithm?

2. How can the supply of electricity and heat demand be integrated into one comprehensive 
evolutionary optimization model?

3. What is the performance of the evolutionary algorithm in regards to each objective relative to an optimization with perfect foresight?

We begin by reviewing relevant literature and then move on to the model and a corresponding case study.

\section{Sector Coupling and Evolutionary Algorithms in Microgrid Implementations}

The existing related literature can be classified into three different streams: (1) microgrid sizing and operation, (2) sector coupling in microgrids and (3) evolutionary algorithms in microgrid optimization.

\subsection{Microgrid Sizing and Operation}

The concept of microgrids has become an active field of research in recent years as it enables the connection and integration of the rising share of decentralized energy resources. Olivares et al. [2] analyse the operational challenges that these complex energy systems create. They point out that advanced control strategies are necessary to coordinate the supply and demand in those decentralized systems, especially if several energy carriers are involved. Zhao et al. [9] see the design and operation of a microgrid as a joint-optimization problem and apply their theory to islanded microgrids in remote geographic areas. To solve their multiobjective sizing and operation problem, Zhao et al. [9] use a method based on a genetic algorithm to find an optimal solution for electricity generation devices in microgrids. Our paper extends the microgrid optimization to the provision of heat for residential households in CECs. Like other authors, Gupta et al. [4] and Berendes et al. [10] acknowledge that the objectives of a community when operating a microgrid are multidimensional and they therefore perform a multiobjective analysis that includes a minimization of emissions and a maximization of self-consumption. Due to the computational complexity, Berendes et al. [10] propose the use of heuristics for microgrid optimization. In their study, Berendes et al. design a software-based tool for sizing and operation of microgrid systems. The open source software tool micrOgridS provides a set of optimal solutions for the configuration and the control of decentralized electricity systems from a multiobjective perspective. In this study, we follow this direction and use an evolutionary algorithm to solve this problem.

\subsection{Sector Coupling in Microgrids}

In this paper, we consider a sector coupled microgrid sometimes also referred to as multi-energy microgrid. Zhao et al. [9], Gupta et al. [4] and Berendes et al. [10] are optimizing the design and operation of microgrids but are solely focusing on electricity, neglecting the demand for heat in their analysis. Zhang et al. [11] introduce micro combined heat and power (mCHP) applications as an effective technology to couple electricity and heat production on a local level. $\mathrm{Gu}$ et al. [12] state that the implementation of cogeneration technologies (e.g. mCHPs) through single applications has several benefits to fulfil energy carrier demands (such as cooling, heating and power). These applications act as reliable sources of electricity generation in microgrids with a high penetration of fluctuating renewable energy from, e.g. photovoltaics systems, which stabilize the supply and increase the overall system efficiency. This paper considers two cogeneration technologies, CHP and photovoltaics thermal (PVT) applications, to fully enable the potential of synergies in parallel heat and power generation. Besides parallel generation, power-to- $x$ technologies play a major role in the development of CECs. Golla et al. [13] use heat pumps to configure optimal CEC setups in which electricity can be used as a resource for heat generation. Alongside battery storage systems, heat pumps can be an efficient application for the utilization of excess electricity either for fulfilling the heat demand directly or using a thermal storage system [14]. Li et al. [15] provide a comprehensive analysis of microgrid operation with multi-energy systems. The implementation of several forms of energy storage (heat storage tank, ice storage tank and battery storage) allows for a high flexibility and an efficient coordination between the energy carriers. In this study, we exploit the full potential of sector coupling in microgrids through the combined implementation of cogeneration and power-to-x technologies as well as energy storage systems for both heat and power. In line with $\mathrm{Gu}$ et al. [12] and Golla et al. [13], this study takes a comprehensive approach and optimizes the sizing and the operation of sector-coupled CECs. This paper extends existing research on sector-coupled operation in microgrids by using an evolutionary algorithm for a multiobjective optimization.

\subsection{Evolutionary Algorithms in Microgrid Optimization}

Using the principle of combining mutation and recombination, evolutionary algorithms (EAs) provide a process of approximating the solution to global 
optimization problems [16]. As outlined in the previous two sections, microgrid operation and sizing can be seen as such a problem with multidimensional objectives. Fadaee et al. [17] reviews research that uses evolutionary algorithms to solve multi-objective optimizations for the control and sizing of microgrids. The authors conclude that heuristic EAs are the most suitable for microgrid optimization. Gupta et al. [4] address the multidimensional objectives of microgrid energy management with a simulation based optimization to identify efficient control strategies that are non-dominated by other strategies. The authors are using the evolutionary computing framework Borg MOEA, which is designed for the optimization of multi-objective, multidimensional problems. The Borg MOEA uses auto-adaptive operators which provide several advantages compared to other MOEAs: identification of search stagnation, avoidance of local optima through randomized restarts and efficient recombination of dominant operators [8].

This study is based on this approach and uses the Borg MOEA framework for an evolutionary multiobjective direct policy search (EMODPS) to determine the optimal application sizing and operation parameters that can be presented to CEC planners and participants.

\section{Enhancing Energy Community Development with EMODPS}

In the following section, the methodology of the proposed EMODPS is introduced. Direct policy search (DPS) is used as a control strategy that searches directly in the policy space [18]. The DPS method parametrizes the policies and reduces the computational complexity when using a simulation-based optimization method. The strategy is particularly suited for problems including multiple objectives because they can be coupled with true MOEAs such as Borg. DPS can be directly coupled with the simulation model and does not add new constraints to the overall structure [19]. The structure of the EMODPS presentation used in this paper is based on the approach proposed by Gupta et al. [4]. First, the conflicting objectives and the general optimization model are explained. Afterwards, we introduce the radial basis functions (RBFs) used to model the operational decisions as well as the variables used to model the sizing of the available technologies. In the last step, the simulation used to model the policy effects and its interaction with the Borg MOEA is explained. For the system structure, different generation, storage and sector coupling technologies are considered. The system is connected to the grid to draw

\begin{tabular}{|c|c|c|}
\hline Variable & Unit & Description \\
\hline$O_{i}$ & & Objective \\
\hline$\Theta$ & & $\begin{array}{l}\text { Set of applications } \theta \\
\text { (BS, TS, PV, PVT, CHP, HP) }\end{array}$ \\
\hline$a_{1}, a_{2}$ & & Phase shifts on $[0,2 \pi]$ \\
\hline$b^{s}$ & {$[\mathrm{kWh} / \mathrm{kW}]$} & Normalized PV generation \\
\hline$c^{e l, g}$ & {$[€ / \mathrm{kWh}]$} & Grid electricity costs \\
\hline$c^{h t, C H P}$ & {$[€ / \mathrm{kWh}]$} & CHP heat costs \\
\hline$c^{i}, c^{i, \theta}$ & $\begin{array}{l}{[€ / \mathrm{kW}]} \\
{[€ / \mathrm{kWh}]}\end{array}$ & Investment costs (for $\theta$ ) \\
\hline $\operatorname{cop}^{H P}$ & & Coefficient of performance HP \\
\hline$e^{\theta}$ & {$[\mathrm{kg}]$} & $\begin{array}{l}\mathrm{CO}_{2} \text { emission factor } \\
\text { for application } \theta\end{array}$ \\
\hline$D^{e l}, D^{h t}$ & {$[\mathrm{kWh}]$} & Total electricity/heat demand \\
\hline$d^{e l, H H}$ & [kWh] & Household electricity demand \\
\hline$d^{e l, H P}$ & [kWh] & HP electricity demand \\
\hline$d^{h t, H H}$ & {$[\mathrm{kWh}]$} & Household heat demand \\
\hline$f^{e l, r}$ & {$[\mathrm{kWh}]$} & $\begin{array}{l}\text { Renewable electricity } \\
\text { fed into the grid }\end{array}$ \\
\hline$f^{e l, c}$ & {$[\mathrm{kWh}]$} & $\begin{array}{l}\text { Conventional electricity } \\
\text { fed into the grid }\end{array}$ \\
\hline$F\left(O_{1}, O_{2}\right)$ & & Objective function \\
\hline$g^{e l}$ & {$[\mathrm{kWh}]$} & $\begin{array}{l}\text { Electricity supplied } \\
\text { by the grid }\end{array}$ \\
\hline$i, j$ & & Counting variables \\
\hline$k^{h t}$ & {$[\mathrm{kWh}]$} & $\begin{array}{l}\text { Heat released into } \\
\text { environment }\end{array}$ \\
\hline$l^{\theta}$ & [years] & Lifetime of application $\theta$ \\
\hline$R B F$ & & Radial Basis function \\
\hline$n^{R B F, \alpha, \beta}$ & & Number of RBFs \\
\hline$r^{e l, c}$ & {$[€ / \mathrm{kWh}]$} & $\begin{array}{l}\text { Feed-in tariff for CHP } \\
\text { electricity }\end{array}$ \\
\hline$r^{e l, r}$ & {$[€ / \mathrm{kWh}]$} & $\begin{array}{l}\text { Feed-in tariff for PVT } \\
\text { and PV electricity }\end{array}$ \\
\hline$s^{\theta}$ & {$[\mathrm{kW}]$, } & Size of application $\theta$ \\
\hline$s^{\theta, \max }$ & $\begin{array}{l}{[\mathrm{kW}]} \\
{[\mathrm{kWh}]}\end{array}$ & $\begin{array}{l}\text { Maximum size } \\
\text { of application } \theta\end{array}$ \\
\hline$t$ & & Current time step \\
\hline$T$ & & Number of time steps \\
\hline$X^{e l}, X^{h t}$ & [kWh] & Total electricity/heat supply \\
\hline$x^{e l, \theta}$ & [kWh] & Electricity generation of $\theta$ \\
\hline$x^{h t, \theta}$ & [kWh] & Heat generation of $\theta$ \\
\hline$\alpha^{B S}, \alpha^{T S}$ & [kWh] & Storage load for BS / TS \\
\hline$\eta^{B S}$ & & Cyclic efficiency of BS \\
\hline$\eta^{T S}$ & & Calendaric efficiency of TS \\
\hline$\lambda^{B S}, \lambda^{T S}$ & {$[\mathrm{kWh}]$} & Storage level of BS / TS \\
\hline$\phi^{\theta}$ & & $\begin{array}{l}\text { Electricity to heat ratio } \\
\text { for } \theta \in(C H P, P V T)\end{array}$ \\
\hline$w, c, r, p$ & & Borg MOEA Parameters \\
\hline
\end{tabular}

Table 1: Nomenclature 
or feed in electricity. Besides PV generation, a hybrid PVT plant is considered for both renewable heat and electricity generation. It has already been shown that the technology has the potential to reduce operational costs in sector coupled scenarios [20]. Besides, a CHP is integrated in the system. A heat pump (HP) possibly enables sector coupling between both the electricity and the heat sector. The option to install a battery storage (BS) or a thermal heat storage (TS) is given. For more information on the functionality of the Borg MOEA, please see [8].

\subsection{Objectives}

To enable a successful energy transition, public acceptance is a key factor [21]. Therefore, it is necessary to include varying CEC participant preferences in the decision making process when determining the system structure and operational strategy of CECs. For the CEC participants, those preferences can be the reduction of carbon emissions, revenue maximization or a high degree of self-sufficiency, among others. In the course of this paper, we focus on two objectives: Costs and carbon emissions. The cost objective is given by:

$$
\begin{aligned}
O_{1}= & c^{i}+\sum_{t=1}^{T}\left(g_{t}^{e l} c^{e l, g}+x_{t}^{h t, C H P} c^{h t, C H P}\right. \\
& \left.-f_{t}^{e l, r} r^{e l, r}-f^{e l, c} r^{e l, c}\right) \\
c^{i}= & \sum_{\theta=1}^{N} \frac{c^{i, \theta} s^{\theta}}{l^{\theta}}
\end{aligned}
$$

The objective is the sum of the installation costs for the different appliances as well as the operating costs for electricity and heat supply. For the calculation of $\mathrm{CO}_{2}$ emissions, both emissions from energy generation and the manufacturing and installation are considered. In the case study, emissions of each appliance are approximated through an emission factor. For CHP, PV, PVT and grid electricity, the emissions are calculated with regard to the amount of energy produced. For the devices used to store or convert energy within the system, BS, TS and HP, the emissions are calculated with regard to the application size. The emission objective for the entire simulation is measured in $\mathrm{kg}$ of $\mathrm{CO}_{2}$ and is given by:

$$
\begin{aligned}
O_{2}= & \left(s^{H P} e^{H P}+s^{B S} e^{B S}+s^{T S} e^{T S}\right) \cdot T \\
& +\sum_{t=1}^{T}\left(g_{t}^{e l} e^{g}+x_{t}^{h t, C H P} e^{C H P}+x_{t}^{e l, P V} e^{P V}\right. \\
& \left.+x_{t}^{e l, P V T} e^{P V T}\right)
\end{aligned}
$$

In our case study, the only operational parameter is the heat pump operation. All other operating decisions are deterministic due to corresponding regulation and are fixed within the simulation. However, the system sizing is subject to optimization as well.

\subsection{Optimization}

For the EMODPS search, both objectives derived in Section 3.1 are optimized. The corresponding optimization problem is formulated below. Both objectives are minimized simultaneously in the objective function:

$$
\min _{x^{h t, H P}, s^{\theta}} F\left(O_{1}, O_{2}\right)
$$

The objective function is minimized with regard to Equations (5) to (14). The first two Equations, (5) and (6), represent the balance constraints for the electricity and heat sector:

$$
\begin{aligned}
\sum_{i=1}^{n^{H H}} d_{i, t}^{e l, H H}= & x_{t}^{e l, P V}+x_{t}^{e l, P V T}+x_{t}^{e l, C H P}-d_{t}^{e l, H P} \\
& +g_{t}^{e l}-f_{t}^{e l, r}-f_{t}^{e l, c}+\alpha_{t}^{B S} \forall t \in T \\
\sum_{i=1}^{n^{H H}} d_{i, t}^{h t, H H}= & x_{t}^{h t, P V T}+x_{t}^{h t, C H P}+x_{t}^{h t, H P}-k_{t}^{h t} \\
& +\alpha_{t}^{T S} \forall t \in T
\end{aligned}
$$

The operation and status of the BS and TS are modeled in Equations (7) and (8):

$$
\begin{aligned}
& \lambda_{t}^{B S}=\lambda_{t-1}^{B S}-\left\{\begin{array}{ll}
\alpha_{t}^{B S} \eta^{b s}, & \alpha_{t}^{B S} \leq 0 \\
\alpha_{t}^{B S}, & \alpha_{t}^{B S}>0
\end{array} \forall t \in T\right. \\
& \lambda_{t}^{T S}=\lambda_{t-1}^{T S} \eta^{T S}-\alpha_{t}^{T S} \forall t \in T
\end{aligned}
$$

Equations (9) and (10) represent the coefficient of performance for the heat pump operation and the heat to power ratio for the PVT system and the CHP. The 
electricity generation of both PV and PVT with regard to the system size is expressed in Equation (11). The limitation of the HP load with regard to the system size is stated in Equation (12), the CHP system size is restricted through Equation (13), the storage level for BS and TS is limited to the respective system size in Equation (14), and the maximum system of BS, HP, PV, PVT and TS sizes are determined in Equation (15):

$$
\begin{aligned}
x_{t}^{h t, H P} & =\operatorname{cop}^{H P} d_{t}^{e l, H P} \forall t \in T \\
x_{t}^{h t, \theta} & =\phi_{t}^{\theta} x^{e l, \theta} \forall \theta \in(P V T, C H P), t \in T \\
x^{e l, \theta} & =b^{s} s^{\theta} \forall \theta \in(P V, P V T) \\
0 & \leq d_{t}^{e l, H P} \leq s^{H P} \forall t \in T \\
0 & \leq s^{h t, C H P} \leq \max \left(x_{t}^{h t, C H P}\right) \forall t \in T \\
0 & \leq \lambda_{t}^{\theta} \leq s^{\theta} \forall \theta \in(B S, T S), t \in T \\
0 & \leq s^{\theta} \leq s^{\theta, \max } \forall \theta \in(B S, H P, P V, P V T
\end{aligned}
$$

\subsection{Policy Formulation}

In the proposed scenario, policies for the heat pump operation and sizing parameters for different system applications are implemented. The sizes of the TS and $\mathrm{BS}$ are set with regard to the maximum storage capacity. The size of the PV and PVT plant is set with regard to the installed peak capacity and the size of the CHP and the HP are set with regard to the maximum heat capacity. While the other appliances are set through policy parameters, the CHP size is determined by the maximum heat demand in the period that persists after the remaining sizing decisions have been taken. This is done to ensure that heat demand can be covered at all times, because otherwise, an oversizing of the CHP would always be beneficial due to feed-in tariffs. The capacity sizing policies are given by:

$$
s^{\theta}=p^{\theta} s^{\theta, \max } \forall \theta \in(P V, P V T, B S, T S, H P)
$$

As proposed by Gupta et al. [4], cubic radial basis functions are implemented for the heat pump operation decisions. Two different types of RBFs are implemented, one with regard to the BS level and one with regard to the TS level. The radial basis functions are given by:

$$
\begin{aligned}
& R B F_{i}^{\alpha}=w_{i}\left(\left|\frac{\lambda_{t}^{B S}-c_{i}}{r_{i}}\right|+x_{t}^{2}+y_{t}^{2}\right)^{3} \forall t, i \\
& R B F_{j}^{\beta}=w_{j}\left(\left|\frac{\lambda_{t}^{T S}-c_{j}}{r_{j}}\right|+x_{t}^{2}+y_{t}^{2}\right)^{3} \forall t, j \\
& x^{H P, h t}=\sum_{i=1}^{n^{R B F, \alpha}} R B F_{i}^{\alpha}+\sum_{j=1}^{n^{R B F, \beta}} R B F_{j}^{\beta}
\end{aligned}
$$

Here, $x$ and $y$ are the cyclic representations of the time of the day with $x_{t}=\sin \left(2 \pi t / 24-a_{1}\right)$ and $y_{t}=\cos \left(2 \pi t / 24-a_{2}\right)$, where $a_{1}$ and $a_{2}$ are the phase shifts on $[0,2 \pi]$. A total of four radial basis functions is used, two considering the BS storage load $\lambda^{B S}$ and two considering the TS storage load $\lambda_{t}^{T S}$ with parameter limits $w_{i} \in[-2,2], c_{i} \in[-2,2], r_{i} \in$ $[-2,2]$. As defined in [4], the goal of EMODPS is to present a non-dominated set of parameters that minimizes the system objectives. In this study, the weights, centres and radii of the RBFs are used to model the HP operation decisions. The inclusion of both the TS and BS storage levels as two different system states enhances previous studies by providing the opportunity to determine operation policies in sector coupled scenarios. Additionally, the sizing parameters offer the ability for local communities to enhance their microgrid by adding new appliances. The sizing parameters are directly included in the objective function and therefore can be set without the use of explicitly modelled RBFs.

\subsection{Simulation and Implementation}

Based on the inputs derived from the policy formulation in Section 3.3, the simulation calculates the objective values and thereby enables an evaluation of the policy. The simulation structure is depicted in Figure 1. The system loads and device operations are calculated in each time step, while the application size is set once for the entire time horizon. The heat and electricity demand of all households are used as input. Based on the application size, the PV plant supplies a given amount of electricity while the PVT plant supplies both heat and electricity. The heat pump operation is determined through policy parameters. TS and BS are operated based on the demand or supply in the respective sector. The CHP is used to cover the remaining heat demand, after heat generation of the HP, PVT and the TS are used. Excess heat cannot be sold, but is instead released into the environment. Electricity produced by the CHP while satisfying the heat demand is consumed 
locally or fed into the grid if demand is lower than generation. Here, the connected grid is both able to supply electricity in times of high demand or absorb electricity from PV, PVT and CHP in times of excess generation. A renewable energy feed-in tariff is paid for fed-in electricity from the PV and PVT panels, a slightly lower feed-in tariff is paid for electricity from the CHP, following current German regulation.

The general structure of the interaction between simulation and the Borg MOEA is displayed in Figure 2. The parameters initially generated by the Borg MOEA are fed into the simulation that returns a set of results for the different objectives that are then reported back to the Borg MOEA. The Borg MOEA uses the information to determine new parameters for the next evaluation using an auto-adaptive multi-operator recombination that is suited for a broad range of problem domains [22]. The algorithm uses an adaptive configuration of simulated binary crossover, differential evolution, parent-centric crossover, unimodal normal distribution crossover, simplex crossover and uniform mutation to determine new parameters for the next evaluation. Aside from uniform mutation, the offspring produced by the other operators is mutated using polynomial mutation. The results of the recombination are then evaluated and considered for inclusion in the archive [8]. All dominated policies, i.e. policies that are outperformed by another policy in all objectives considered are discarded. After the final round of evaluation, the DPS system returns a set of non-dominated policies.

The system is able to determine both the application size of the considered technologies and parameters for a corresponding operation policy. The returned set of non-dominated solutions can be used to enable the decision-making process of local community participants based on individual preferences ${ }^{1}$.

\section{Case Study}

To demonstrate the functionality of the EMODPS, we apply the Borg MOEA and the simulation model described in Section 3.4 to one year of data including residential heat and electricity consumption in hourly resolution of ten households with an average of 3,286 $\mathrm{kWh}$ electricity demand and 15,237 $\mathrm{kWh}$ heat demand per year. The individual load profiles are created using the load profile generator by Pflugradt et al. [23]. The solar generation data is simulated for a CEC located in southern Germany. To show the algorithm performance with regard to different seasons, we evaluate three scenarios: 'summer','winter' and

\footnotetext{
${ }^{1}$ The entire simulation code is available at: https://github.com/ArminGo/HICSSBorg
}

\begin{tabular}{|c|c|c|c|c|}
\hline \multirow[t]{2}{*}{ Application } & \multicolumn{2}{|c|}{$\begin{array}{l}\text { Investment } \\
\text { costs }\end{array}$} & Lifetime & $\begin{array}{l}\mathrm{CO}_{2} \\
\text { emissions }\end{array}$ \\
\hline & \multicolumn{2}{|c|}{$[€ / \mathrm{kWh}]$} & [years] & {$[\mathrm{kg} / \mathrm{kWh}]$} \\
\hline CHP [24] & \multicolumn{2}{|c|}{1700} & 20 & $0.207 *$ \\
\hline PV [25] & \multicolumn{2}{|c|}{1400} & 20 & $0.050 *$ \\
\hline PVT $[25,26]$ & \multicolumn{2}{|c|}{1800} & 20 & $0.055^{*}$ \\
\hline BS [13] & \multicolumn{2}{|c|}{1700} & 20 & $83.5 * *$ \\
\hline TS $[27,28]$ & \multicolumn{2}{|c|}{40} & 20 & $12 * *$ \\
\hline HP $[13,29]$ & \multicolumn{2}{|c|}{1450} & 17 & $1060 * *$ \\
\hline \multicolumn{5}{|c|}{$\begin{array}{l}* \mathrm{~kg} \mathrm{CO}_{2} / \mathrm{kWh} \text { production } * * \mathrm{~kg} \mathrm{CO}_{2} / \mathrm{kWh} \text { system size } \\
\text { CHP: Combined Heat and Power, PV: Photovoltaic, } \\
\text { PVT: PV/Thermal, BS: Battery Storage, } \\
\text { TS: Thermal Storage, HP: Heat Pump }\end{array}$} \\
\hline Tariff & & Costs & $\mathrm{CO}_{2} \mathrm{emi}$ & sions \\
\hline$g^{e l}[30]$ & & 0.30 & 0.40 & \\
\hline$x^{h t, C H P}$ & [24] & 0.10 & 0.20 & \\
\hline$f^{e l, r}$ & & 0.10 & 0.00 & \\
\hline$f^{e l, c}$ & & 0.08 & - & \\
\hline
\end{tabular}

Table 2: Investment costs, $\mathrm{CO}_{2}$ emissions and lifetime for specific technologies

'mid-season'. For the summer scenario, the household load and solar generation data between May and August is aggregated to an average week to reduce the necessary computation time. For the winter scenario, we use the data for the months from November to February and the mid-season scenario includes March, April, September and October. The system configuration for investment costs and $\mathrm{CO}_{2}$-emissions is based on the situation in the German energy market. An overview of the technology parameters for this study is given in Table 2. Energy generation costs, grid charges and feed-in tariffs are also based on German regulation and also shown in Table 2. For the analysis, each scenario is run with 30 initial sets of randomly chosen parameters over 200,000 evaluation rounds by the Borg MOEA (Version 1.9). The simulation is carried out over the averaged week for each scenario. For comparison, each scenario is also analyzed with linear optimization models that regard each objective individually.

\subsection{Results of the EMODPS}

The results indicate a wide range of non-dominated policies that can be used for the sizing and operation of the considered system. Figure 3 shows the non-dominated policies for each initial seed for the summer scenario. Each point represents one 


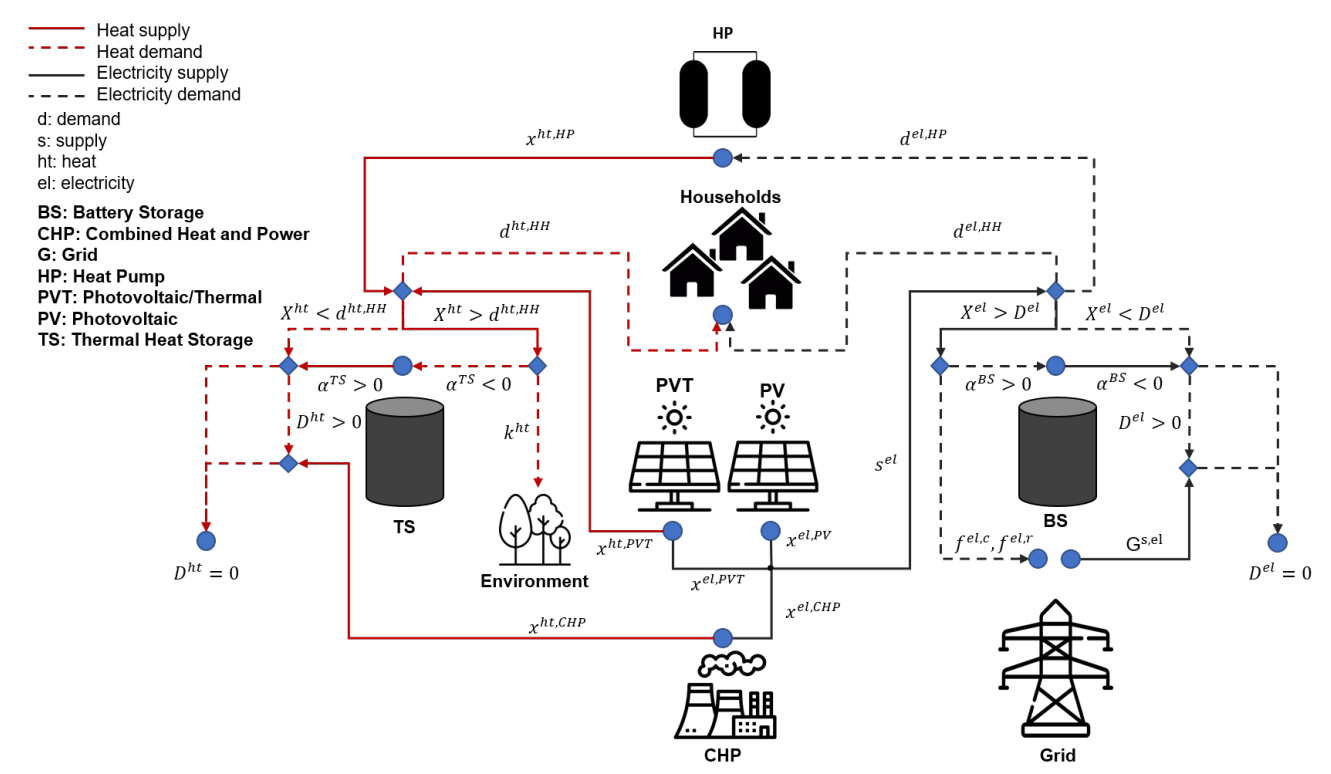

Figure 1: Simulation Flowchart.

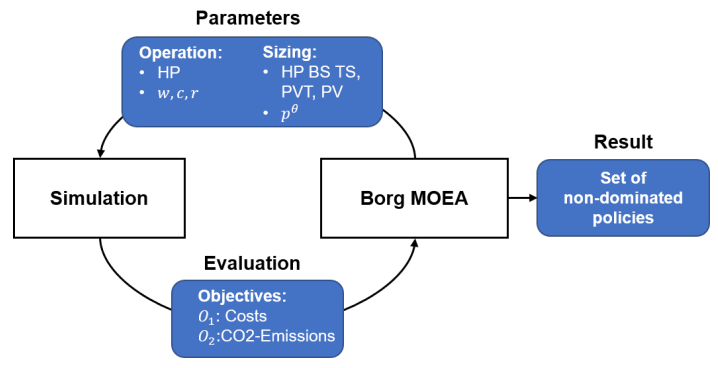

Figure 2: Interaction between the simulation and the Borg MOEA for the EMODPS

non-dominated result for a sizing and operation policy. As to be expected, the DPS for the summer scenario returns policies with the lowest overall costs compared to other scenarios. That can be explained by the high PV and PVT generation potential compared to low heating costs in that period. The gap in the pareto front exists due to a change between two general strategies in the application sizing decisions for the summer scenario. In Figure 4, each line represents the sizing decisions of one DPS solution with regard to the application sizes relative to their maximum installation sizes. The two strategies mentioned mainly differ in the sizing of the BS and the TS. The DPS solutions that achieve better results for the cost objective favor both large PV and PVT systems, indicating that the current feed-in tariff policy in Germany incentivises the installation of residential solar PV. The linear optimizations for the summer scenario return costs of $€ 26$ for $O_{1}$ and $43 \mathrm{~kg} \mathrm{CO}_{2}$

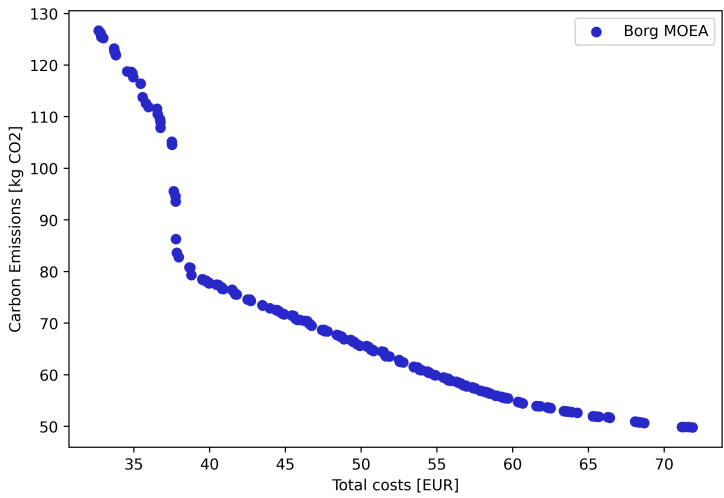

Figure 3: Pareto front for the summer scenario

emissions for $\mathrm{O}_{2}$ as optimal individual solutions. The DPS solutions for the mid-season scenario presented in Figure 5 result in both higher costs and emissions for a mixed strategy than the summer scenario. Figure 6 shows that the policies mainly differ in the sizing of the PV plant, while all other parameters remain similar. The larger TS system in comparison to both the summer scenario and the winter scenario indicate a higher volatility in the heat demand, as the mid-season scenario already includes days with higher heating demand. For renewable generation, the PVT plant is favored over the PV system with regard to ecological interests. The linear optimization for the mid-season scenario returns costs of $€ 117.5$ for $O_{1}$ and $111.7 \mathrm{~kg} \mathrm{CO}_{2}$ emissions for $O_{2}$ in the individual optimization. The difference between the optimization and the simulation results shows that the 


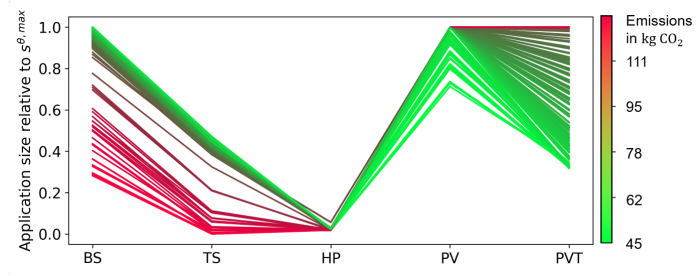

Figure 4: Application sizing decisions for the summer scenario

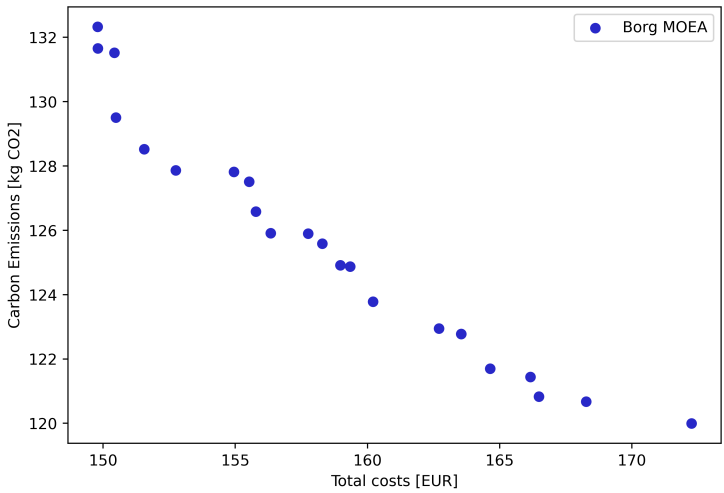

Figure 5: Pareto front for the mid-season scenario

action space needs to be more thoroughly searched for the mid-season scenario which might be explained by the difference between spring and fall.

The results for the EMODPS in the winter scenario return the highest costs and emissions, as depicted in Figure 7. Recommendations for the HP size implementation in the winter scenario are distinctly larger then in the other scenarios, but do not exhaust the maximum application size available, as can be seen in Figure 8. The PVT plant is again built to the maximum size for all DPS solutions, while the size of the PV plant correlates with with the policy emissions. The linear optimization for the winter scenario returns costs of $€ 285$ for $O_{1}$ and $371 \mathrm{~kg} \mathrm{CO}$ emissions for $O_{2}$ as individual optima.

The insights provided by the EMODPS on an

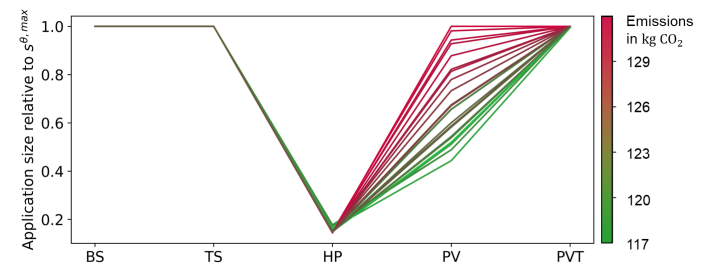

Figure 6: Application sizing decisions for the mid-season scenario

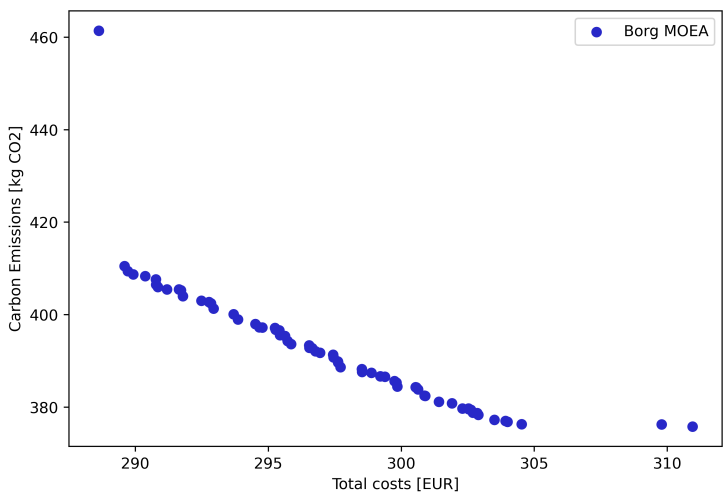

Figure 7: Pareto front for the winter scenario

individual level for each scenario show the different policies that can be used to plan and operate a CEC. Depending on the season in focus, the DPS recommends different strategies and sizing decisions, although some parameters, like a large PVT plant, are recommended through all scenarios. The following section provides further evaluation of the results with variable parameters.

\subsection{Sensitivity Analysis}

Besides the HP operation parameters, the simulation results are very dependent on the application sizing decisions determined by the Borg MOEA. In the in-between scenario, PVT and BS are built to the maximum size for all policies, resulting in a $25 \mathrm{~kW}$ PVT plant and a $30 \mathrm{kWh}$ BS. To demonstrate the effect of these two system components on the overall outcome for the energy community, the input sizes for the simulation configurations are varied for one exemplary policy in the mid-season scenario with costs of $€ 156$ and $\mathrm{CO}_{2}$ emissions of $127 \mathrm{~kg}$ for the one week period. The operation parameters for the heat pump and all sizing decisions, except for BS and PVT remain constant. The $\mathrm{BS}$ and PVT size is then varied between 1 and $60 \mathrm{kWh}$ and $\mathrm{kW}$. The results for the cost objective are displayed in Figure 9. While an increased size of the PVT plant

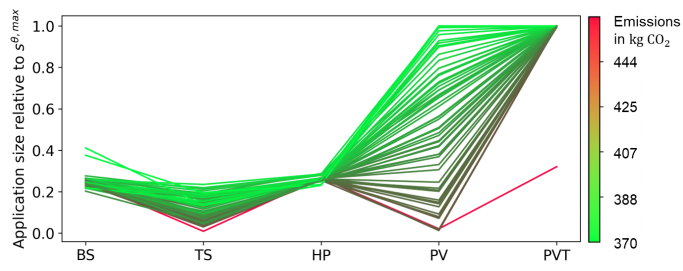

Figure 8: Application sizing decisions for the winter scenario 


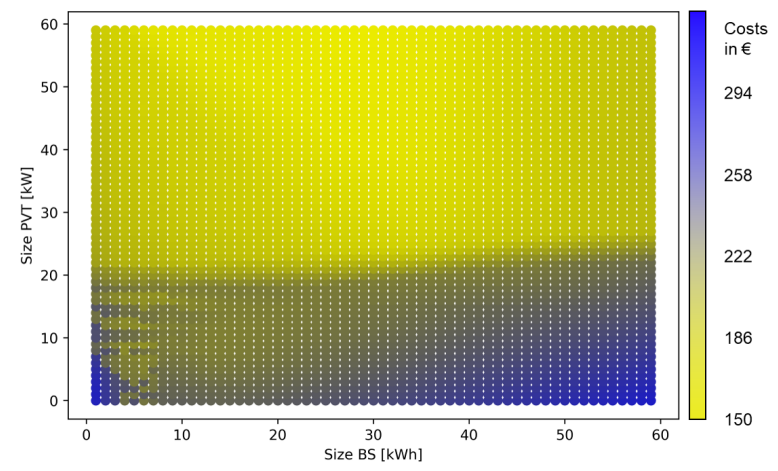

Figure 9: Sensitivity analysis of the cost objective with regard to different system sizes

generally leads to reduced costs, the effect of the BS size depends on the size of the PVT. For a small PVT, a high BS size increases overall costs, while a smaller BS is able to utilize excess energy generated by the PVT plant. Figure 10 shows the same variations with regard to the emission objective. Here, the turquoise section shows the positive network effects of a simultaneous increase of BS and PVT. A larger BS enables the use of generated renewable electricity instead of using electricity from the grid with a higher emission factor.

\section{Discussion and Outlook}

The results of the case study indicate the potential of EMODPS for the operation and sizing of sector-coupled CECs. With DPS, the interested parties can see the effects of different preference selections before needing to specifically state these preferences. Future work needs to investigate group decision processes to select one individual policy based on individual preferences of participants, investors and operators in a CEC. While a general setup for a decision support system has already

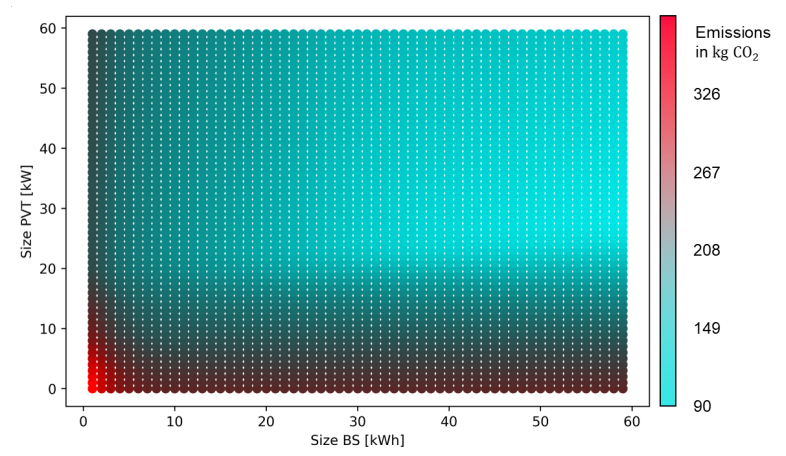

Figure 10: Sensitivity analysis of the emission objective with regard to different system sizes been provided [13], the exact specifications of this system remain subject to further research. With regard to the emission objective, this work focuses on $\mathrm{CO}_{2}$ emissions that arise through investment and generation within the community and through generation supplied by the grid that is used in the community, represented through the grid emission factor in the case study. The potential emission benefits of feeding in low emission electricity generation from PV, PVT and CHP into the grid and the associated system emission reductions are not considered. As a whole, CECs with renewable generation have the ability to lower emissions system wide and a system perspective is therefore an important next step. The constrained application sizes in the case study are given as fixed values, as larger appliances require more space and the application size is therefore limited. While some applications are used with the maximum size available in each scenario, namely the PV and PVT panel, other applications like the HP are only used at a fraction of the maximum size available. In reality, technologies like PV and PVT often have competing maximum application sizes for example due to limited roof space. Future research should incorporate joint sizing options for all available applications as interdependent decisions.

\section{Conclusion}

The model for evolutionary, multi-objective, direct policy search in the context of development and operation of Citizen Energy Communities presented in this paper enables the integrated assessment of operation and application sizing decisions with regard to competing objectives. The model integrates both the demand for heat and electricity on a community level. The policies regard both the thermal storage and the battery storage level to model the heat pump operation and are within range of a linear optimization of each individual objective. The set of solutions can be used to enable local decision makers in energy communities to develop their community based on individual preferences. Participants, investors and local operators are able to see the effects of their installation decisions and the energy costs and emissions for the community. The model proposed in this paper can be integrated into a decision support system that helps residents to become energy communities and build their own renewable generation technologies. We aim to bridge the interest gap between investors, local residents and energy suppliers and thereby contribute to a successful, decentralized energy transition. 


\section{References}

[1] G. A. Barzegkar-Ntovom, N. G. Chatzigeorgiou, A. I. Nousdilis, S. A. Vomva, G. C. Kryonidis, E. O. Kontis, G. E. Georghiou, G. C. Christoforidis, and G. K. Papagiannis, "Assessing the viability of battery energy storage systems coupled with photovoltaics under a pure self-consumption scheme," Renewable Energy, vol. 152, pp. 1302-1309, 2020

[2] D. E. Olivares, A. Mehrizi-Sani, A. H. Etemadi, C. A Cañizares, R. Iravani, M. Kazerani, A. H. Hajimiragha, O. Gomis-Bellmunt, M. Saeedifard, R. Palma-Behnke, et al., "Trends in microgrid control," IEEE Transactions on smart grid, vol. 5, no. 4, pp. 1905-1919, 2014.

[3] A. Baldinelli, L. Barelli, G. Bidini, and G. Discepoli, "Economics of innovative high capacity-to-power energy storage technologies pointing at $100 \%$ renewable micro-grids," Journal of Energy Storage, vol. 28, p. 101198,2020

[4] A. Gupta, M. Liu, D. Gold, P. Reed, and C. L. Anderson, "Exploring a direct policy search framework for multiobjective optimization of a microgrid energy management system," in Proceedings of the 53rd Hawaii International Conference on System Sciences, 2020.

[5] T. A. Nguyen and M. Crow, "Stochastic optimization of renewable-based microgrid operation incorporating battery operating cost," IEEE Transactions on Power Systems, vol. 31, no. 3, pp. 2289-2296, 2015.

[6] H. Karimi and S. Jadid, "Optimal microgrid operation scheduling by a novel hybrid multi-objective and multi-attribute decision-making framework," Energy, vol. 186, p. 115912, 2019.

[7] M. M. Sokołowski, "Renewable and citizen energy communities in the european union: how (not) to regulate community energy in national laws and policies," Journal of Energy \& Natural Resources Law, pp. 1-16, 2020.

[8] D. Hadka and P. Reed, "Borg: an auto-adaptive many-objective evolutionary computing framework," Evolutionary computation, vol. 21, no. 2, pp. 231-259, 2013.

[9] B. Zhao, X. Zhang, P. Li, K. Wang, M. Xue, and C. Wang, "Optimal sizing, operating strategy and operational experience of a stand-alone microgrid on dongfushan island," Applied Energy, vol. 113, pp. 1656 - 1666, 2014.

[10] S. Berendes, P. Bertheau, and P. Blechinger, "Sizing and optimization of hybrid mini-grids with microgrids-an open-source modelling tool," 072018.

[11] D. Zhang, S. Evangelisti, P. Lettieri, and L. G. Papageorgiou, "Optimal design of chp-based microgrids: Multiobjective optimisation and life cycle assessment," Energy, vol. 85, pp. 181 - 193, 2015.

[12] W. Gu, Z. Wu, R. Bo, W. Liu, G. Zhou, W. Chen, and $\mathrm{Z}$. Wu, "Modeling, planning and optimal energy management of combined cooling, heating and power microgrid: A review," International Journal of Electrical Power \& Energy Systems, vol. 54, pp. 26 - 37, 2014.

[13] A. Golla, S. Henni, and P. Staudt, "Scaling the concept of citizen energy communities through a platform-based decision support system," European Conference on Information Systems (ECIS), 2020.
[14] A. Golla, J. Geis, T. Loy, P. Staudt, and C. Weinhardt, "An operational strategy for district heating networks: application of data-driven heat load forecasts," Energy Informatics, vol. 3, no. S1, 2020.

[15] Z. Li and Y. Xu, "Optimal coordinated energy dispatch of a multi-energy microgrid in grid-connected and islanded modes," Applied Energy, vol. 210, pp. 974 - 986, 2018.

[16] T. Bäck, Evolutionary Algorithms in Theory and Practice: Evolution Strategies, Evolutionary Programming, Genetic Algorithms. Oxford University Press, Inc., 1996.

[17] M. Fadaee and M. Radzi, "Multi-objective optimization of a stand-alone hybrid renewable energy system by using evolutionary algorithms: A review," Renewable and Sustainable Energy Reviews, vol. 16, no. 5, pp. 3364 $-3369,2012$.

[18] Verena Heidrich-Meisner and Christian Igel, "Hoeffding and bernstein races for selecting policies in evolutionary direct policy search," International Conference on Machine Learning, Montreal, Canada, vol. 26, 2009.

[19] M. Giuliani, A. Castelletti, F. Pianosi, E. Mason, and P. M. Reed, "Curses, tradeoffs, and scalable management: Advancing evolutionary multiobjective direct policy search to improve water reservoir operations," Journal of Water Resources Planning and Management, vol. 142, no. 2, p. 04015050, 2016.

[20] A. Golla, P. Staudt, and C. Weinhardt, "Combining pvt generation and air conditioning: A cost analysis of surplus heat utilization," International Conference on Smart Energy Systems and Technologies, 2019.

[21] P. Staudt, A. Golla, B. Richter, M. Schmidt, F. vom Scheidt, and C. Weinhardt, "Behavioral studies in energy economics: A review and research framework," 42nd IAEE International Conference, 2019.

[22] D. Hadka and P. Reed, "Diagnostic assessment of search controls and failure modes in many-objective evolutionary optimization," Evolutionary computation, vol. 20, no. 3, pp. 423-452, 2012.

[23] N. Pflugradt, J. Teucher, B. Platzer, and W. Schufft, "Analysing low-voltage grids using a behaviour based load profile generator," Renewable Energy and Power Quality Journal, pp. 361-365, 2013.

[24] H. Falkenberg, B. Eikmeier, S. Gores, M. Gailfuß, and O. Antoni, "Evaluierung der kraft-wärme-kopplung: Analysen zur entwicklung der kraft-wärmekopplung in einem energiesystem mit hohem anteil erneuerbarer energien," 2019.

[25] H. Wirth, "Aktuelle fakten zur photovoltaik in deutschland," 2020.

[26] T. Lauf, M. Memmler, and S. Schneider, "Emissionsbilanz erneuerbarer energieträger: Bestimmung der vermiedenen emissionen im jahr 2018," 2019.

[27] A. Thess, F. Trieb, A. Wörner, and S. Zunft, "Herausforderung wärmespeicher," Physik Journal, vol. 14, pp. 33-39, 2015.

[28] F. Samweber and C. Schifflechner, "Kostenanalyse wärmespeicher bis 10.0001 speichergröße," 2016.

[29] E. P. Johnson, "Air-source heat pump carbon footprints: Hfc impacts and comparison to other heat sources," Energy Policy, vol. 39, no. 3, pp. 1369-1381, 2011.

[30] P. Icha and G. Kuhs, "Entwicklung der spezifischen kohlendioxidemissionen des deutschen strommix in den jahren 1990 - 2019,” 2019. 